\title{
Диффузионное размытие квантовых ям GaAs, выращенных при низкой температуре
}

\author{
(C) В.И. Ушанов ${ }^{1}$, В.В. Чалдышев ${ }^{1}$, В.В. Преображенский ${ }^{2}$, М.А. Путято ${ }^{2}$, Б.Р. Семягин ${ }^{2}$ \\ ${ }^{1}$ Физико-технический институт им. А.Ф. Иоффре Российской академии наук, \\ 194021 Санкт-Петербург, Россия \\ ${ }^{2}$ Институт физики полупроводников Сибирского отделения Российской академии наук, \\ 630090 Новосибирск, Россия \\ E-mail: Decorus2009@mail.ru, chald.gvg@mail.ioffe.ru
}

(Получена 31 мая 2018 г. Принята к печати 6 июня 2018 г.)

Методом фотолюминесцентной спектроскопии исследованы процессы диффузионного размытия периодической системы квантовых ям GaAs, разделенных барьерами AlGaAs, выращенной методом молекулярнолучевой эпитаксии при низкой температуре $\left(200^{\circ} \mathrm{C}\right)$ и дополнительно легированной изовалентными примесями $\mathrm{Sb}$ и Р. Отжиг при температуре $750^{\circ} \mathrm{C}$ в течение 30 мин после роста приводил к увеличению энергии пика фотолюминесценции экситонного состояния $e 1-h h 1$ в квантовых ямах вследствие размытия эпитаксиальных интерфейсов $\mathrm{GaAs} / \mathrm{AlGaAs}$, вызванных усиленной взаимодиффузией атомов $\mathrm{Al}-\mathrm{Ga}$ по катионной подрешетке. Для профиля концентрации Al в квантовых ямах, определяемого линейной диффузией, было решено уравнение Шредингера для электронов и дырок. Оказалось, что экспериментально наблюдаемое энергетическое положение пика фотолюминесценции соответствует длине взаимной диффузии $\mathrm{Al}-\mathrm{Ga} 3.4$ нм и эффективному коэффициенту диффузии $6.3 \cdot 10^{-17} \mathrm{~cm}^{2} / \mathrm{c}$ для температуры $750^{\circ} \mathrm{C}$. Полученное значение оказалось близким к значению для квантовых ям GaAs, выращенных при низкой температуре без дополнительного легирования примесями $\mathrm{Sb}$ и Р. Полученные результаты позволяют сделать вывод о незначительном влиянии усиленной взаимодиффузии $\mathrm{As}-\mathrm{Sb}$ и $\mathrm{As}-\mathrm{P}$ в анионной подрешетке на процессы взаимодиффузии $\mathrm{Al}-\mathrm{Ga}$ по катионной подрешетке.

DOI: $10.21883 /$ FTP.2018.13.46873.8920

\section{1. Введение}

Разработка полупроводниковых гетероструктур и сверхрешеток с интерфейсами высокого кристаллического качества стала возможной благодаря развитию технологии молекулярно-лучевой эпитаксии (МЛЭ), использование которой при низких температурах роста позволяет дополнительно сформировать новый класс материалов, обладающих высоким быстродействием при сохранении качества эпитаксиальных гетероинтерфейсов. Так, для приборных приложений большой интерес представляют наноструктуры на основе GaAs, выращенного методом МЛЭ при низкой температуре (LT-GaAs), времена жизни носителей в котором ультракороткие и могут достигать субпикосекундных значений [1-4]. Так, в работе [5] была исследована система квантовых ям (КЯ) LT-(GaAs/AlAs), выращенных методом МЛЭ при низкой температуре. Время жизни носителей заряда в такой системе составило 15 пс. В данной работе авторами было впервые показано, что материалы на основе LT-GaAs, обладающие ультракороткими временами жизни носителей, одновременно могут иметь в оптических спектрах узкие экситонные особенности, обусловленные резкими интерфейсами квантовых ям. Данные свойства делают наноструктуры на основе LT-GaAs перспективными для использования в насыщающихся поглотителях, фоторефрактивных устройствах, детекторах ближнего инфракрасного излучения, а также для электрооптического сэмплирования [6].
Эффективность полупроводниковых приборов на основе гетероструктур, выращенных методом МЛЭ, напрямую определяется качеством полученных гетеропереходов, а также их морфологией. Большую роль при дальнейшей термообработке играют размытие и композиционное перемешивание интерфейсов вследствие наличия примесных атомов и избыточных антиструктурных дефектов [1-3,7-10]. В работе [2] было показано существенное влияние послеростового отжига при температурах $>700^{\circ} \mathrm{C}$ на оптические и электронные свойства системы квантовых ям на основе LT-GaAs и барьеров LT-AlAs. Высокая температура отжига приводила к усиленной взаимодиффузии атомов $\mathrm{Al}-\mathrm{Ga}$, что вызывало размытие и огрубление эпитаксиальных интерфейсов GaAs/AlAs, приводя к смещению и деградации изначально резких экситонных особенностей в оптических спектрах.

Процессы взаимодиффузии в наноструктурах, выращенных методом МЛЭ, также интенсивно исследовались методами просвечивающей электронной микроскопии (ПЭМ) [8-11], рентгеновской дифракции [12], сканирующей туннельной микроскопии [13] и моделировались методами Монте-Карло [14].

Отклонение от стандартных режимов эпитаксиального роста, в частности понижение температуры подложек $\mathrm{GaAs}$, обеспечивает сильную нестехиометричность получаемых эпитаксиальных пленок, обусловленную высокой концентрацией антиструктурных дефектов [15]. Исследования, проводимые для нестехиометрических гетероструктур LT-(GaAs/InAs) [8,9] и LT-(GaAs/AlAs) [1-3], 
показали наличие значительной взаимодиффузии атомов $\mathrm{In}-\mathrm{Ga}$ и $\mathrm{Al}-\mathrm{Ga}$ соответственно по катионной подрешетке GaAs. Основной причиной такой усиленной диффузии была высокая концентрация вакансий галлия $\left(V_{\mathrm{Ga}}\right)[16]$, значение которой в LT-GaAs может достигать $10^{18} \mathrm{~cm}^{-3}$, что существенно больше соответствующей величины в стехиометрическом материале.

Основные точечные дефекты (атомы замещения, $\mathrm{As}_{\mathrm{Ga}}$, и вакансии галлия, $\mathrm{V}_{\mathrm{Ga}}$ ), связываемые с LT-GaAs, однако, не вносят вклада во взаимодиффузию, протекающую по анионной подрешетке [10,17]. Поскольку диффузия по подрешетке элементов V группы определяется механизмом замещения межузельных атомов $\mathrm{As}_{i}$ в $\mathrm{GaAs}$, она должна быть чувствительна к концентрации $\mathrm{As}_{i}$. В работе [10] исследовались процессы диффузионного перемешивания по мышьяковой подрешетке в GaAs, выращенном методом МЛЭ при низкой температуре и подвергнутом периодическому $\delta$-легированию изовалентной примесью $\mathrm{Sb}$. Исследования выращенных наноструктур методами ПЭМ выявили существенное размытие $\delta$-слоев $\mathrm{Sb}$, сопровождавшееся формированием металлических преципитатов $\mathrm{AsSb}$ вследствие послеростового высокотемпературного отжига. Замещающая диффузия $\mathrm{As}-\mathrm{Sb}$ по анионной подрешетке за счет межузельных атомов $\mathrm{As}_{i}$ оказалась значительно усиленной в LT-GaAs. Оценка концентрации межузельных атомов $\mathrm{As}_{i}$ составила $(3-6) \cdot 10^{18} \mathrm{~cm}^{-3}$, что сопоставимо с величиной концентрации вакансий галлия в LT-GaAs, обеспечивающих взаимодиффузию по катионной подрешетке.

Исследование механизмов усиленной взаимодиффузии в LT-GaAs по одной из подрешеток при наличии усиленной диффузии по смежной подрешетке вследствие легирования материала, однако, изучено мало. В настоящей работе нами исследовалась периодическая система LT-(GaAs/AlGaAs), подвергнутая легированию примесями $\mathrm{Sb}$ и $\mathrm{P}$, и рассмотрены процессы взаимодиффузии $\mathrm{Al}-\mathrm{Ga}$ по катионной подрешетке при наличии замещающей диффузии $\mathrm{As}-\mathrm{Sb}$ и $\mathrm{As}-\mathrm{P}$ по анионной подрешетке LT-GaAs.

\section{2. Образцы и методика эксперимента}

В данной работе методом МЛЭ были выращены два образца на подложке полуизолирующего GaAs c ориентацией (001). Образцы представляли собой упорядоченную систему КЯ GaAs толщиной 14 нм и барьеров $\mathrm{AlGaAs}$ толщиной 140 нм. Концентрация AlAs в барьерах составляла $30 \%$, что обеспечивало окно прозрачности для света с длинами волн от 670 нм. $\mathrm{B}$ процессе роста КЯ GaAs подвергались $\delta$-легированию примесью $\mathrm{Sb}$, а барьеры $\mathrm{AlGaAs}$ - примесями $\mathrm{Sb}$ и $\mathrm{P}$. Толщина $\delta$-слоев была 1 монослой. Первый образец (ST1519) был выращен при температуре $T_{\mathrm{gr}}=480^{\circ} \mathrm{C}$, содержал 15 периодов GaAs/AlGaAs и являлся референтным, тогда как второй образец (ST1520) содержал 6 периодов и был выращен при низкой температуре
$\left(T_{\mathrm{gr}}=200^{\circ} \mathrm{C}\right)$, что обеспечивало значительную концентрацию точечных дефектов в объеме эпитаксиальных слоев. Данный образец был разделен на 5 частей, 3 из которых были подвергнуты послеростовому отжигу при температурах 400, 500 и $600^{\circ} \mathrm{C}$ (ST1520-4, ST1520-5 и ST1520-6 соответственно) в течение 5 мин. Последняя часть образца была отожжена при температуре $T_{\text {ann }}=750^{\circ} \mathrm{C}(\mathrm{ST} 1520-7)$ в течение 30 мин.

Оптическое исследование выращенных образцов осуществлялось методом фотолюминесценции (ФЛ) при возбуждении твердотельным лазером KLM-532-х с длиной волны излучения 532 нм при р-поляризации лазерного излучения. Измерения ФЛ проводились при температуре $4.2 \mathrm{~K}$. Регистрация спектров осуществлялась при помощи спектрометра OceanOptics QE65 Pro.

\section{3. Экспериментальные результаты и обсуждение}

Спектры ФЛ при температуре $4.2 \mathrm{~K}$ для референтного образца ST1519 и образца ST1520-7, отожженного при температуре $T_{\mathrm{ann}}=750^{\circ} \mathrm{C}$ в течение 30 мин, показаны на рисунке. Стрелками при энергии 1.519 эВ отмечен край фундаментального поглощения в GaAs (ширина запрещенной зоны $E_{g}$ ) при $4.2 \mathrm{~K}$. Для референтного образца ST1519 при энергии 1.532 эВ наблюдается пик, отвечающий ФЛ в КЯ GaAs, соответствующей основному экситонному состоянию для тяжелых дырок, $e 1-h h 1$. Для образца, выращенного при низкой температуре и подвергнутого дальнейшей термообработке, аналогичный пик смещается в область больших энергий и

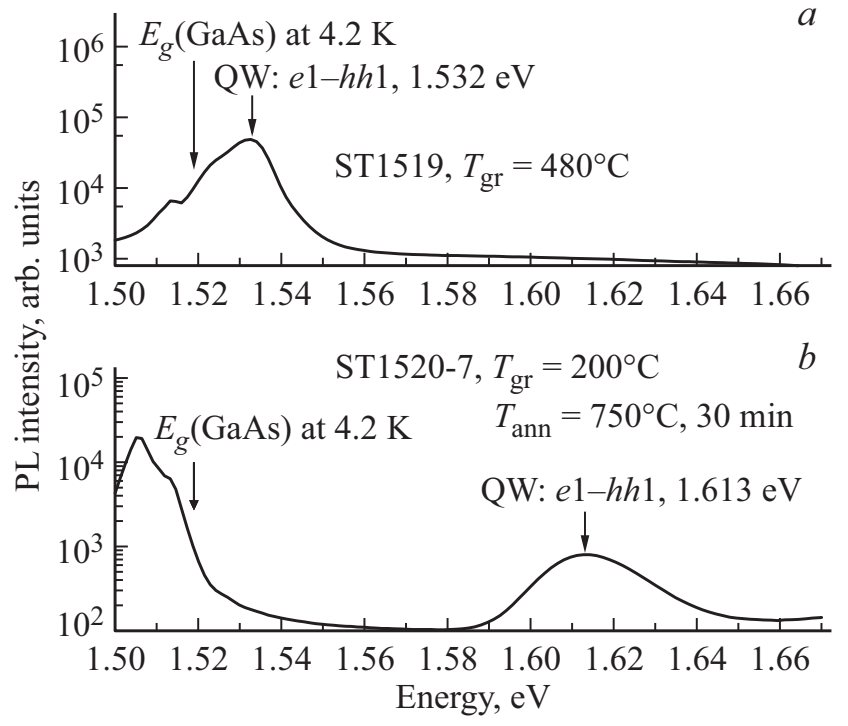

Спектры фотолюминесценции (PL) при $4.2 \mathrm{~K}$ референтного образца и образца, выращенного при низкой температуре $\left(200^{\circ} \mathrm{C}\right)$, затем отожженного при температуре $750^{\circ} \mathrm{C}$ в течение 30 мин. Стрелками отмечены особенности, связанные с краем фундаментального поглощения в GaAs, а также пики, соответствующие переходам $e 1-h h 1$ в квантовой яме (QW) LT-GaAs. 
наблюдается при значении энергии 1.613 эВ, при этом наблюдаемая интенсивность пика уменьшается практически в 60 раз. Ненулевой уровень регистрируемого сигнала вдали от резонансных особенностей вызван фоновой засветкой при рассеянии лазерного излучения в криостате. Особенности, связанные с ФЛ в КЯ GaAs для образцов, выращенных при низкой температуре и отожженных при температурах $400-600^{\circ} \mathrm{C}$ в течение 5 мин, в эксперименте обнаружены не были.

Наблюдаемое значительное увеличение энергии пика ФЛ для перехода $e 1-h h 1$ в образце ST1520-7 относительно референтного может быть обусловлено увеличением концентрации Al в КЯ LT-GaAs вследствие диффузии атомов Al из барьеров LT- AlGaAs [2]. Модификация геометрии КЯ из-за размытия интерфейсов также ведет к изменению энергии основного перехода $e 1-h h 1$. $\mathrm{B}$ режиме линейной диффузии профиль концентрации Al в КЯ, центр которой расположен в начале координат, может быть описан с помощью дополнительной функции ошибок [2]:

$$
C(x)=\frac{1}{2}\left[\operatorname{erfc}\left(\frac{x+b / 2}{2 L_{D}}\right)-\operatorname{erfc}\left(\frac{x-b / 2}{2 L_{D}}\right)\right]+1 .
$$

где $x$ - координата, $b$ - ширина КЯ, $L_{D}-$ длина диффузии. В данном уравнении в качестве единственного подгоночного параметра выступала длина диффузии, тогда как остальные параметры были фиксированы и определялись условиями роста. Несмотря на то что формирование профиля концентрации $\mathrm{Al}$ в общем случае может зависеть от значения концентрации $\mathrm{Al}$ в данной точке, ожидается, что такие отклонения от линейного режима малы [2]. Диффузия атомов $\mathrm{Al}$ в область КЯ также модифицирует профиль энергетического потенциала $V$ и профили эффективных масс $m^{*}$ электронов, тяжелых и легких дырок [18]. Уровни энергии электронов и дырок в размытой КЯ могут быть получены решением уравнения Шредингера с соответствующими зависимостями $V[C(x)]$ и $m^{*}[C(x)]$ :

$$
\left[-\frac{\hbar^{2}}{2 m^{*}(x)} \nabla^{2}+V(x)\right] \psi(x)=E \psi(x) .
$$

Решение данного уравнения осуществлялось подбором значения длины диффузии, обеспечивающего необходимый профиль диффузионного размытия КЯ так, что расчетная энергия перехода $e 1-h h 1$ соответствовала экспериментальному значению 1.613 эВ с поправкой на энергию связи экситона 8 мэВ. Полученное таким образом значение длины диффузии составило $3.4 \mathrm{HM}$, что позволяет сделать оценку величины эффективного коэффициента взаимодиффузии $\mathrm{Al}-\mathrm{Ga}$ с учетом времени послеростовой термообработки образца $t_{\mathrm{ann}}$ :

$$
D_{\mathrm{eff}}=\frac{L_{D}^{2}}{t_{\mathrm{ann}}}=6.3 \cdot 10^{-17} \mathrm{~cm}^{2} / \mathrm{c} .
$$

Данное значение существенно превышает величину коэффициента диффузии для стехиометрического GaAs, что явно свидетельствует об усиленной взаимодиффузии $\mathrm{Al}-\mathrm{Ga}$ по катионной подрешетке в рассматриваемой наноструктуре.

Процессы диффузионного перемешивания интерфейсов КЯ GaAs/AlGaAs, выращенных методом МЛЭ при низкой температуре и не подвергавшихся дополнительному легированию примесями элементов III или $\mathrm{V}$ групп, рассматривались в работе [2]. Полученные аналитические зависимости эффективного коэффициента взаимодиффузии $\mathrm{Al}-\mathrm{Ga}$ позволили оценить его величину для отжига при температуре $750^{\circ} \mathrm{C}$ как $6.5 \cdot 10^{-17} \mathrm{~cm}^{2} / \mathrm{c}$, что близко к значению, полученному в настоящем исследовании. Аналогичное исследование процессов взаимодиффузии по катионной подрешетке в LT-GaAs, выращенном методом МЛЭ, осуществлялось в работах $[8,9]$ для атомов In и Ga. Исследование микроструктуры $\delta$-слоев In в LT-GaAs методом ПЭМ показало существенное размытие индийсодержащих слоёв, обусловленное взаимодиффузией атомов In-Ga, усиленной наличием высокой концентрации вакансий галлия. По измерениям толщин индийсодержащих слоев при различных температурах послеростового отжига были получены зависимости для эффективного коэффициента взаимодиффузии In-Ga, величина которого составила $21.4 \cdot 10^{-17} \mathrm{~cm}^{2} / \mathrm{c}$ для температуры отжига $750^{\circ} \mathrm{C}$.

В указанных работах легирование образцов, обеспечивающее усиленную взаимодиффузию атомов As по анионной подрешетке, не проводилось и процессы диффузии по мышьяковой подрешетке GaAs не рассматривались. В работе [10] было показано, что взаимодиффузия по анионной подрешетке $\mathrm{As}-\mathrm{Sb}$ в LT-GaAs, периодически $\delta$-легированном $\mathrm{Sb}$, оказалась значительно усиленной. Значение коэффициента диффузии для температуры отжига $750^{\circ} \mathrm{C}$, согласно [10], составляло $1.8 \cdot 10^{-17} \mathrm{~cm}^{2} / \mathrm{c}$, что сопоставимо с величиной эффективного коэффициента диффузии по катионной подрешетке. Полученное в данной работе значение для эффективного коэффициента взаимодиффузии $\mathrm{Al}-\mathrm{Ga}\left(D_{\mathrm{Al}-\mathrm{Ga}}\right)$ по катионной подрешетке оказывается близким к соответствующему значению в нелегированном LT-GaAs из работы [2]. Данный факт свидетельствует о незначительном влиянии процессов замещающей диффузии $\mathrm{As}-\mathrm{Sb}$ и As-P по анионной подрешетке в исследуемой системе LT-(GaAs/AlGaAs) на взаимодиффузию атомов Al-Ga в галлиевой подрешетке.

\section{4. Заключение}

Таким образом, в данной работе нами исследована упорядоченная система КЯ LT-GaAs и барьеров LT-AlGaAs, выращенных методом МЛЭ и подвергнутых $\delta$-легированию изовалентными примесями $\mathrm{Sb}$ и $\mathrm{P}$. Экспериментальное исследование спектров ФЛ при температуре $4.2 \mathrm{~K}$ показало наличие пиков, связанных с ФЛ экситонных состояний $e 1-h h 1$ в КЯ LT-GaAs, для референтного образца, выращенного при нормальной температуре, а также для образца, выращенного при 
низкой температуре и отожжённого при $750^{\circ} \mathrm{C}$ в течение 30 мин. В последнем случае пик ФЛ для состояния $e 1-h h 1$ имел сдвиг в область больших энергий на 81 мэВ, что было связано со значительным размытием и композиционным перемешиванием интерфейсов исследуемых КЯ LT-GaAs. Подгоночное значение длины диффузии для оценки профиля концентрации Al в КЯ LT-GaAs составило 3.4 нм, что также позволило оценить величину эффективного коэффициента взаимодиффузии $\mathrm{Al}-\mathrm{Ga}$ в катионной подрешетке, $6.3 \cdot 10^{-17} \mathrm{~cm}^{2} / \mathrm{c}$. Полученное значение оказалось близким к значению для LT-GaAs в случае отсутствия легирования примесями $\mathrm{Sb}$ и $\mathrm{P}$, участвующими в замещающей диффузии по анионной подрешетке. Данное наблюдение указывает на то, что взаимодиффузия $\mathrm{As}-\mathrm{Sb}$ и $\mathrm{As}-\mathrm{P}$ в анионной подрешетке оказывает слабое влияние на взаимодиффузию $\mathrm{Al}-\mathrm{Ga}$ в катионной подрешетке, ответственную за размытие профиля исследуемых КЯ LT-GaAs.

Работа выполнена при поддержке программы президиума РАН „Актуальные проблемы фотоники, зондирование неоднородных сред и материалов“ и Российского фонда фундаментальных исследований (грант № 17-02-01168).

\section{Список литературы}

[1] I. Lahiri, D.D. Nolte, M.R. Melloch, J.M. Woodall, W. Walukiewicz. Appl. Phys. Lett., 69, 239 (1996).

[2] R. Guersen, I. Lahiri, M. Dinu, M.R. Melloch, D.D. Nolte. Phys. Rev. B, 60, 10926 (1999).

[3] I. Lahiri, D.D. Nolte, J.C.P. Chang, J.M. Woodall, M.R. Melloch. Appl. Phys. Lett., 67, 1244 (1995).

[4] А.А. Пастор, У.В. Прохорова, П.Ю. Сердобинцев, В.В. Чалдышев, М.А. Яговкина. ФТП, 47, 8 (2013).

[5] I. Lahiri, D.D. Nolte, E.S. Harmon, M.R. Melloch, J.M. Woodall. Appl. Phys. Lett., 66, 2519 (1995).

[6] D.D. Nolte. J. Appl. Phys., 85, 6259 (1999).

[7] D.G. Deppe, N. Holonyak. J. Appl. Phys., 64, R93 (1988).

[8] N.A. Bert, V.V. Chaldyshev, Yu.G. Musikhin, A.A. Suvorova, V.V. Preobrazhenskii, M.A. Putyato, B.R. Semyagin, P. Werner. Appl. Phys. Lett., 74, 1442 (1999).

[9] Н.А. Берт, Ю.Г. Мусихин, В.В. Преображенский, М.А. Путято, Б.Р. Семягин, А.А. Суворова, В.В. Чалдышев, P. Werner. ФТП, 32, 769 (1998).

[10] V.V. Chaldyshev, N.A. Bert, Yu.G. Musikhin, A.A. Suvorova, V.V. Preobrazhenskii, M.A. Putyato, B.R. Semyagin, P. Werner, U. Gösele. Appl. Phys. Lett., 79, 1294 (2001).

[11] H. Bender, W. Coene, A.F.D. Jong. Ultramicroscopy, 21, 373 (1987).

[12] D.B. Mc Whan, R.M. Fleming, A.C. Gossard, W. Wiegmann, R.A. Logan. J. Appl. Phys., 51, 357 (1980).

[13] D.A. Collins, R.M. Feenstra, D.Z.Y. Ting, M.W. Wang, T.C. Mc Gill. Phys. Rev. Lett., 72, 2749 (1994).

[14] J. Singh, K.K. Bajaj. Appl. Phys. Lett., 47, 594 (1985).

[15] V.V. Chaldyshev. Mater. Sci. Eng. B, 88, 195 (2002).

[16] T. Tan, U. Goesele, S. Yu. Crit. Rev. Solid State Mater. Sci., 17, 47 (1991).
[17] M. Schultz, U. Egger, R. Scholz, O. Breitenstein, U. Gösele, T.Y. Tan. J. Appl. Phys., 83, 5295 (1998).

[18] S. Adachi. J. Appl. Phys., 58, R1 (1985).

Редактор Л.В. Шаронова

\section{Diffusive intermixing of GaAs quantum wells grown at low temperature}

\author{
V.I. Ushanov ${ }^{1}$, V.V. Chaldyshev ${ }^{1}$, \\ V.V. Preobrazhenskii ${ }^{2}$, M.A. Putyato ${ }^{2}$, B.R. Semyagin ${ }^{2}$ \\ ${ }^{1}$ loffe Institute, \\ 194021 St. Petersburg, Russia \\ ${ }^{2}$ Institute of Semiconductor Physics, \\ Siberian Branch of Russian Academy of Sciences, \\ 630090 Novosibirsk,Russia
}

\begin{abstract}
By using the photoluminescence spectroscopy we studied a diffusive intermixing in a periodic system of GaAs quantum wells and $\mathrm{AlGaAs}$ barriers grown by molecular beam epitaxy at low temperature $\left(200^{\circ} \mathrm{C}\right)$ and doped with isovalent $\mathrm{Sb}$ and $\mathrm{P}$ impurities. A post-growth annealing at the temperature of $750^{\circ} \mathrm{C}$ for $30 \mathrm{~min}$ resulted in an increase of the photoluminescence peak energy for the $e 1-h h 1$ exciton state in the quantum wells due to the compositional intermixing at the GaAs/AlGaAs interfaces caused by the enhanced $\mathrm{Al}-\mathrm{Ga}$ interdiffusion in the cation sublattice. Schröedinger equation for electrons and holes was solved for the $\mathrm{Al}$ concentration profile in the quantum wells, which was determined by linear diffusion. Experimentally observed energy of the photoluminescence peak appeared to correspond to an $\mathrm{Al}-\mathrm{Ga}$ interdiffusion length of $3.4 \mathrm{~nm}$ and effective diffusion coefficient of $6.5 \cdot 10^{-17} \mathrm{~cm}^{2} / \mathrm{s}$ for the annealing temperature of $750^{\circ} \mathrm{C}$. The obtained value turned out to be close to the corresponding value for the GaAs quantum wells grown at low temperature without additional doping with $\mathrm{Sb}$ and $\mathrm{P}$ impurities. The obtained results allowed us to conclude that the enhanced interdiffusion of $\mathrm{As}-\mathrm{Sb}$ and $\mathrm{As}-\mathrm{P}$ atoms in the anion sublattice had no significant effects on the $\mathrm{Al}-\mathrm{Ga}$ interdiffusion in the cation sublattice.
\end{abstract}

